\title{
Wer definiert die Medizin?
}

Die heutige Medizin funktioniert nach Prämissen, die sie innerhalb ihrer Fachgrenzen kaum reflektiert. Wie wichtig eine philosophisch geleitete Diskussion dieser Prämissen wäre, macht die Ökonomisierung der Medizin deutlich, die politisch gefordert wird und zugleich Unbehagen in der Praxis verbreitet. Solche Widersprüche deuten für das Selbstverständnis der Medizin auf Bedarf an Neudefinitionen. Die Philosophie stellt hierfür Methoden und Konzepte bereit.

Enno Rudolph ${ }^{a}$,

Manuel Bachmann ${ }^{b}$

a Prof. Dr., Ordinarius für Philosophie, Leiter des Kulturwissenschaftlichen Instituts und wissenschaftlicher Gesamtleiter des neuen Weiterbildungsprogramms «Philosophie und Medizin», Universität Luzern

b Dr. phil., MBA HSG, Studienleiter des Weiterbildungsprogramms «Philosophie und Medizin», Universität Luzern
Korrespondenz:

Dr. phil. Manuel Bachmann

Universität Luzern

Kasernenplatz 3

CH-6000 Luzern 7

manuel.bachmann@unilu.ch

\section{Der Spielraum des Arztes unter dem Diktat der Effizienz}

Effizienzanforderungen schränken den Spielraum ärztlichen Handelns immer mehr ein. Der Arzt wird übersteuert vom Case-Manager. Das Gesundheitswesen soll als produktives System funktionieren. Dessen Leistung wird quantifiziert und mittels Benchmarking kontrollierbar gemacht. Entscheidungen sollen nicht nur problemorientiert, sondern auch profitorientiert ausfallen. Im Gegenzug scheint der ärztliche Spielraum mit dem wissenschaftlichen Fortschritt zu wachsen. Dieser dehnt die Grenzen des Machbaren unaufhaltsam aus. Indessen, wer entscheidet - und nach welchen Kriterien -, was im Einzelfall an teuren Technologien und neuen Therapien einzusetzen sinnvoll und geboten ist?

Die Steigerung des medizinisch Machbaren spitzt diese Frage zu. An der Forschungsfront der Medizin wird zunehmend über die Zukunft der Bewältigung solcher Krankheiten entschieden, die bislang als Schicksal hingenommen wurden. Hier wird allerdings nicht nur definitiv über gesund und krank entschieden, sondern das jeweilige Urteil wird indirekt durch vorgeschaltete Entscheidungen über «geeignet/ungeeignet» bzw. «nützlich/unnütz» gesteuert. Diese Entscheidungen spiegeln gesellschaftliche Erwartungen und unterliegen den Kriterien des ökonomischen Fortschritts.

Damit schliesst sich der Kreis, der den ärztlichen Entscheidungsspielraum zunehmend verringert: Wenn die Entscheidungskriterien vom ökonomischen Fortschritt vorgegeben werden, folgt auch der medizinische Fortschritt dem Diktat der Effizienz.

\section{Es geht um Definitionsmacht}

Diesem Problem sieht sich jede praktizierende Ärztin bzw. jeder Arzt ausgeliefert. Wenn es um die Frage nach medizinischer Professionalität geht, geht es um die Frage nach der Definitionsmacht über das Selbstverständnis der Medizin: Wer definiert die Medizin? In der ökonomisierten Medizin spiegelt sich die faktische Definitionsmacht ökonomischer Interessen im
Zusammenspiel mit gesellschaftlichen Erwartungen und wissenschaftlichem Fortschritt.

Diese Frage ist heute unausweichlich geworden und markiert einen neutralen und grundsätzlichen Standpunkt philosophischer Reflexion moderner Medizin. Die Philosophie ist die akademische Disziplin, die hierzu die erforderlichen Instrumente bereitstellt. Philosophische Methoden und Konzepte ermöglichen, Prinzipien, Kategorien und Denkvoraussetzungen der Medizin systematisch zu untersuchen. Eine solche philosophische Reflexion kann sich mit der Deskription der faktischen Definitionsmacht denn auch nicht lange aufhalten. Sie muss vordringen zur Frage: Wie kann und wie soll die Medizin angemessen definiert werden? Aus den denkbaren Antworten ergeben sich die praktischen Konsequenzen: von der Medizinethik über die Forschungspolitik bis zur konkreten Gestaltung des medizinischen Versorgungssystems. Insofern ist diese Frage nicht nur für die medizinischen Praktiker, sondern für alle Akteure im Gesundheitswesen interessant und entscheidend.

\section{Erforderliche Neudefinitionen}

Selbstverständnis, Grundbegriffe und Grenzfragen der Medizin sind heute durch Forschungsdynamik, technologische Innovation und ethische Probleme chaotisch konfiguriert. In dieser unübersichtlichen Situation rächen sich einseitige Blickwinkel, seien sie politisch oder fachwissenschaftlich festgelegt. So führt beispielsweise das konsequente Benchmarking in der Spitalplanung zu paradoxen Ergebnissen, indem rein quantitative Benchmarks festgelegt werden, die Qualitätseinbussen in der medizinischen Leistungserbringung belohnen. Ähnlich entzieht sich einer quantifizierenden Sichtweise ein fundamentaler Baustein ärztlicher Professionalität: das vertrauensbildende Gespräch zwischen Arzt und Patient. Weitere Beispiele für abgekürztes Denken in der medizinischen Praxis liessen sich anführen. Sie alle sind allerdings nicht als Einzelprobleme relevant, sondern als Symptome philosophischer Definitionsdefizite grundlegender Art. 
Herausgegriffen seien drei Problemstellungen, welche die philosophische Tragweite der erforderlichen Definitionen sichtbar machen:

1. Wie kann ökonomische Effizienz mit ärztlicher Autonomie und sozialer Solidarität korreliert werden? Durch Rationierung medizinischer Leistung entstehen Interessenkonflikte und Interventionsparadoxien. Die markt- und profitorientierte Forschung bewirkt ökonomisch bedingte Verzerrungen medizinischer Evidenz. Die Institutionen des Gesundheitswesens müssen das Bedürfnis nach Autonomie von Arzt und Patient berücksichtigen. Das dabei zu erreichende Mass an Effizienz muss so festgelegt werden, dass auch Solidarität in der medizinischen Versorgung gewährleistet ist. Die Philosophie stellt hierfür ökonomisch fundierte Gesellschafts-, Freiheits- und Gerechtigkeitsmodelle bereit.
Mediziners konkret und zeitgemäss zu entwerfen. Die Philosophie liefert Identitätskriterien für den guten Arzt bzw. die gute Ärztin - nicht abstrakt und abgehoben, sondern in einem ständigen Gespräch mit der praktischen Medizin, indem sie deren begriffliche und kulturelle Voraussetzungen diskussionsfähig macht. Auf diese Weise hilft die philosophische Reflexion der Medizin, eine neue Definitionsmacht über ihr Selbstverständnis zu gewinnen.

\section{Verlangt die Medizin nach einer neuen Anthropologie?}

Wie unverzichtbar die philosophische Reflexion auf das Selbstverständnis der Medizin ist, wird abschliessend an der Frage deutlich, ob die Medizin nach einer neuen Anthropologie, einer Neudefinition des Menschen verlangt. Philosophisch ist zwischen humanistischer und naturalistischer Anthropologie zu unter-

\section{Philosophische Methoden und Konzepte ermöglichen es, die Prinzipien und Denkvoraussetzungen der Medizin systematisch zu untersuchen}

2. Wie sind die Kategorien «Gesundheit/Krankheit» zu definieren?

Bereits die Geschichte der Medizin lehrt, wie relativ die Zuordnungen «gesund» bzw. «krank» in unterschiedlichen historischen und kulturellen Kontexten ausfallen. So fundamental und funktional diese Kategorien für die ärztliche Praxis sind, so deutungsoffen stellen sie sich einer kritischen Überprüfung dar. Wo endet die Therapie einer Krankheit, wo beginnt das Human Enhancement? Auch die evidenzbasierte Medizin benutzt in Diagnoseprozessen nur Wahrmacher, die von einer solchen relativen Kategorienbasis abhängen. Diese Basis zu reflektieren, ist eine Aufgabe, die in einem philosophischen Rahmen vorzüglich geleistet werden kann.

3. Wer entscheidet nach welchen Kriterien den Grenzfall?

Wer übernimmt wofür Verantwortung? Die Medizinethik ist bereits per definitionem eine philosophische Disziplin. Sie muss sich auf neue Fragen einstellen, die der medizinische Fortschritt generiert. Das kann sie allerdings nur, wenn sie von den Fachwissenschaften nicht vereinnahmt wird. Sie muss ein philosophisches Reflexionsniveau halten, indem sie ethisch relevante Strukturtypen ärztlichen Handelns identifiziert und die Wirkung medizinischer Intervention verbindet mit prinzipiellen Definitionen ärztlicher Verantwortung.

\section{Was macht den guten Arzt aus?}

Solche Neudefinitionen dienen einem praktischen Zweck. Sie stellen Kriterien bereit, das Berufsbild des scheiden. Letztere unterstellt, dass der Mensch hinreichend und abschliessend als reines Naturwesen verstanden werden muss. Die erregten Debatten über die Frage, ob die jüngeren Forschungsresultate in der Neurophysiologie das Reden von Geist, Seele, Bewusstsein oder Freiheit überflüssig gemacht haben oder nicht, sind ein Indiz für eine Tendenz in eine Richtung, die auf einen medizinisch legitimierten Sieg des Naturalismus als Definitionsmacht in den Lebenswissenschaften hinweist. Vor diesem Hintergrund erwacht ein starkes Interesse seitens vieler Vertreter der medizinischen Wissenschaften wie auch seitens der medizinischen Praxis an den Antworten der hier zuständigen Wissenschaft: Es ist die Philosophie, die weder zulässt, den Menschen ausschliesslich auf seine körperliche Materie zu reduzieren, noch die Akteure in der medizinischen Theorie und Praxis von der Verantwortung für das menschliche Individuum dispensiert.

Der neue berufsbegleitende Nachdiplomkurs «Philosophie und Medizin» der Universität Luzern richtet sich an Spezialärzte und Allgemeinpraktiker, an Spitalkader und im Gesundheitswesen tätige Fachleute. Das Programm mit philosophisch und medizinisch qualifizierten Hochschuldozenten umfasst 12 Kurstage und beginnt am 2. Dezember 2010. Anmeldeschluss ist der 1. November 2010. Weitere Informationen zum Studiengang finden sich unter: www.philomedizin.ch 